\title{
CIUDADES INTERMEDIAS HERRAMIENTA LOCAL CON VISION GLOBAL. Caso de Estudio del Area Metropolitana de Mérida. Estado Mérida - Venezuela.
}

\section{INTERMEDIATE CITIES LOCAL TOOL WITH GLOBAL $\mathrm{VISION}$ \\ Case Study of the Metropolitan Area of Merida. State of Merida - Venezuela.}

\section{Leyda Coromoto Rondón Velazquez*}

RESUMEN: Las ciudades intermedias son urbes de tamaño mediano y pequeñas, con condiciones específicas, que le permiten interactuar mejor con las áreas rurales, sin embargo, en la actualidad en estos espacios se promueve la expansión territorial, que generan problemas medio ambientales y estimulan un crecimiento acelerado, fragmentado y disperso, transformándolos en asentamientos grandes e ineficientes. En función de esta realidad se persigue, como objetivo, "definir un mecanismo de planificación en donde se propone interactuar eficientemente, entre lo urbano y lo rural, entre lo regional y la ciudad, haciendo de esta manera sostenible su ordenamiento y desarrollo deseado, con la finalidad de que dicho instrumento pueda ser implantado sobre el Área Metropolitana de Mérida, Venezuela". El estudio, se logró mediante el desarrollo teórico del concepto de la ciudad intermedia y la identificación de sus características, junto a un mecanismo de planeación que ordena y permite el desarrollo eficiente y sostenible de ambos espacios, logrando así una mejor cohesión del territorio. Como resultado, se plantea un equilibrio entre lo económico, lo social y lo ambiental, a través de la planificación territorial estratégica, con ventajas para desarrollar mejor la sostenibilidad en las ciudades intermedias, en donde las áreas rurales y urbanas, van a interactuar en forma armónica, permitiendo una mejor calidad de vida, a través de una comprensión del medio ambiente y el medio construido.

Palabras clave: Ciudades intermedias, Desarrollo Sostenible, Peri-urbanización, Planificación Estratégica Territorial

Recibido 18 de noviembre 2020/aceptado el 12 de noviembre 2021

* Red Internacional de Promotores de los Objetivos de Desarrollo Sostenible RIPO CHILE leydarondon@gmail.com https:/ / orcid.org/0000-0003-1096-5423 
ABSTRACT: Intermediate cities are small and medium-sized cities, with specific conditions that allow them to interact better with rural areas; however, at present, these spaces promote territorial expansion, which generates environmental problems and stimulates accelerated, fragmented and dispersed growth, transforming them into large and inefficient settlements; In light of this reality, the objective is to "define a planning mechanism, where it is proposed to interact efficiently between the urban and the rural, between the regional and the city, thus making its planning and desired development sustainable, with the purpose that this instrument can be implemented in the Metropolitan Area of Merida, Venezuela". The study was achieved through the theoretical development of the concept of the intermediate city and the identification of its characteristics, together with a planning mechanism that organizes and allows the efficient and sustainable development of both spaces, thus achieving a better cohesion of the territory. As a result, a balance between economic, social and environmental aspects is proposed, through strategic territorial planning, with advantages to better develop sustainability in intermediate cities, where rural and urban areas will interact harmoniously, allowing a better quality of life, through an understanding of the environment and the built environment.

Key words: Intermediate cities, Sustainable Development, Peri-urbanization, Strategic Territorial Planning.

\section{INTRODUCCIÓN}

El rápido crecimiento urbano que se originó a principio del siglo XX, después de la revolución m industrial, creó una nueva organización con formas e intensidades diferentes, junto a una concentración acelerada de la población, situación que da origen a las ciudades que tenemos hoy en día, donde se generan regiones que visualizan dos espacios diferentes, el campo y la ciudad, produciéndose a la vez, zonas de transición entre una y otra, bajo condiciones muy similares, llamada periferia, esa línea delgada que no es reconocida con facilidad, pero que define la separación entre una y la otra, donde la urbanización es fragmentada, dinámica entre ambos lados y donde se produce el cambio entre lo regional y lo produce el cambio entre lo regional y lo urbano, de una manera muy similar, con elementos de carácter económico, territorial y de planeación, propias de las ciudades llamadas intermedias, espacios que resultarían más eficientes y sostenibles, si se analizara ambas escalas al mismo tiempo. (Llop, 2013, p. 34).

Si estos espacios, campo y ciudad, se desarrollaran bajo una relación que los interconectara e interrelacionara entre sí y que a la vez generarán cambios en la forma en que las percibimos, podríamos llegar a disminuir o limitar el crecimiento de las ciudades, dando una visión diferente del campo, que ayudaría a mejorar el tipo de urbanización que se estimula en la ciudad.

Debido a la pujante migración campo-ciudad, la mayoría de la población mundial en la 
actualidad se encuentra ocupando espacios urbanos, incrementando estas áreas en comparación con el resto del territorio, situación que permite identificar las diferentes ciudades que se han derivado de este proceso, que van desde los pequeños centros poblados o asentamientos, hasta las grandes aglomeraciones, derivadas de la unión de varias áreas metropolitanas, denominadas Mega ciudades 0 Megalópolis, estando en medio de ellas las urbes, con poblaciones intermedias, donde se logran obtener espacios eficientes, interactuando en forma diferente con su entorno; es por ello, que este trabajo propone comprobar ¿̇con qué mecanismos se puede desarrollar una mejor cohesión sobre el territorio más sostenible?, con el fin de lograr una mejor calidad de vida dentro de conurbaciones similares al área de estudio.

Las ciudades son asentamientos que se caracterizan por abarcar grandes extensiones de suelo, sin embargo, existen grupos que se concentran en espacios más pequeños y otros que se desarrollan utilizando grandes espacios urbanos, donde todos persiguen el mismo objetivo, el de satisfacer necesidades, buscar mejores servicios y desarrollar una mejor calidad de vida. La definición de las ciudades llamadas intermedias varía según las especificidades, leyes o reglamentos de cada país, pudiéndose centrar en criterios tanto cuantitativos como cualitativos de lo que puede ser ese tipo de espacio (Roig, 2014 , p. 44-46).

Derivado de este planteamiento, se persigue como objetivo "definir un mecanismo de planificación, en donde se propone interactuar eficientemente, entre lo urbano y lo rural, entre lo regional y la ciudad, haciendo de esta manera sostenible su ordenamiento y desarrollo deseado, con la finalidad de que dicho mecanismo pueda ser implantado sobre el área de estudio del área Metropolitana de Mérida-Ejido-Tabay ubicado en Venezuela".

\section{DESCRIPCIÓN DEL ÁREA DE ESTUDIO:}

El área de estudio se ubica sobre el área metropolitana de Mérida, capital del estado Mérida (Figuras 1 y 2), en Venezuela, compuesta por tres municipios: Libertador (Mérida), Campo Elías (Ejido) y Santos Marquina (Tabay), donde se estima una población de 508.988 habitantes (según Censo 2000). 


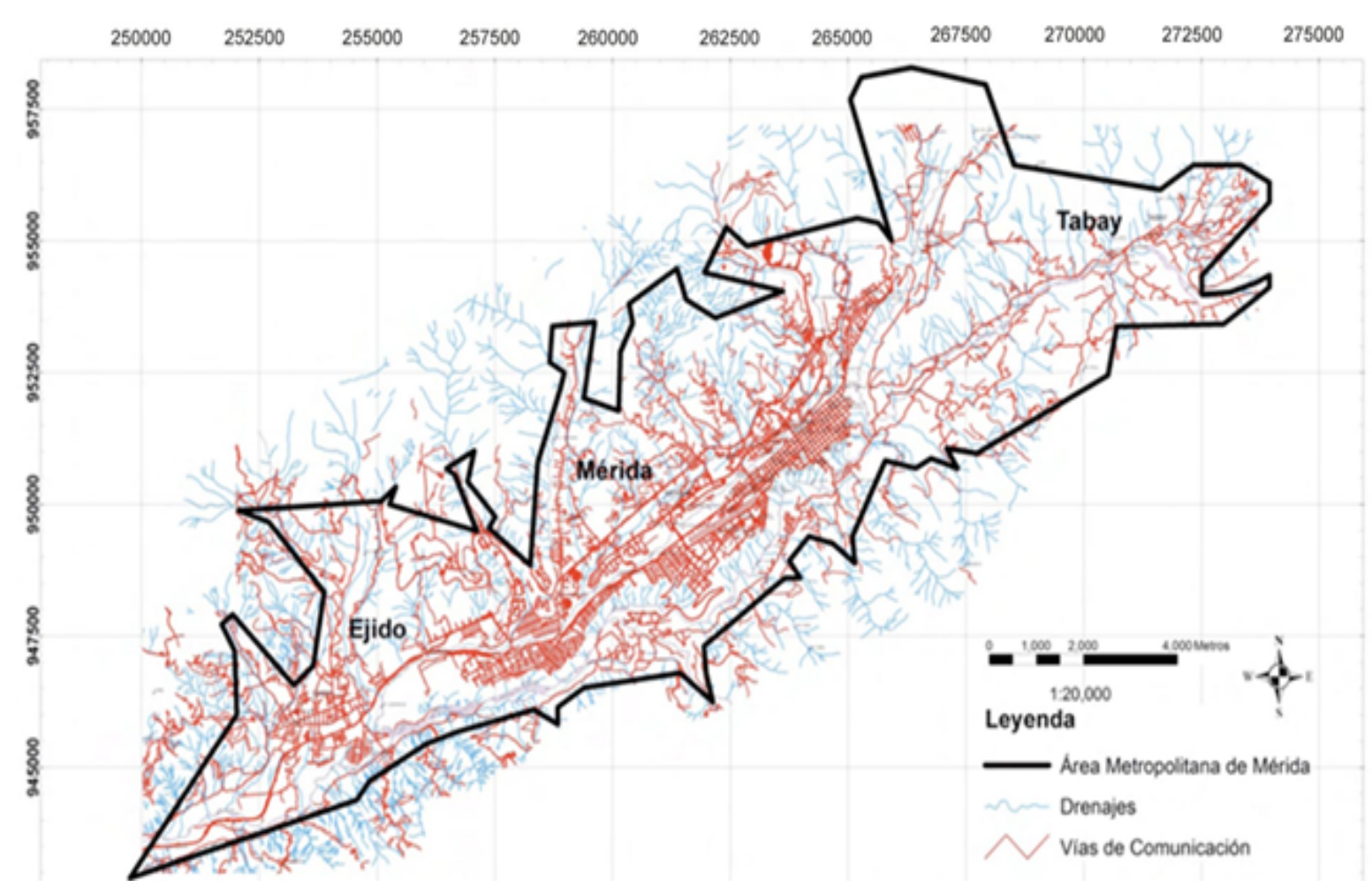

Figura 1. Poligonal Urbana del Área Metropolitana de Mérida. Fuente: elaboración propia

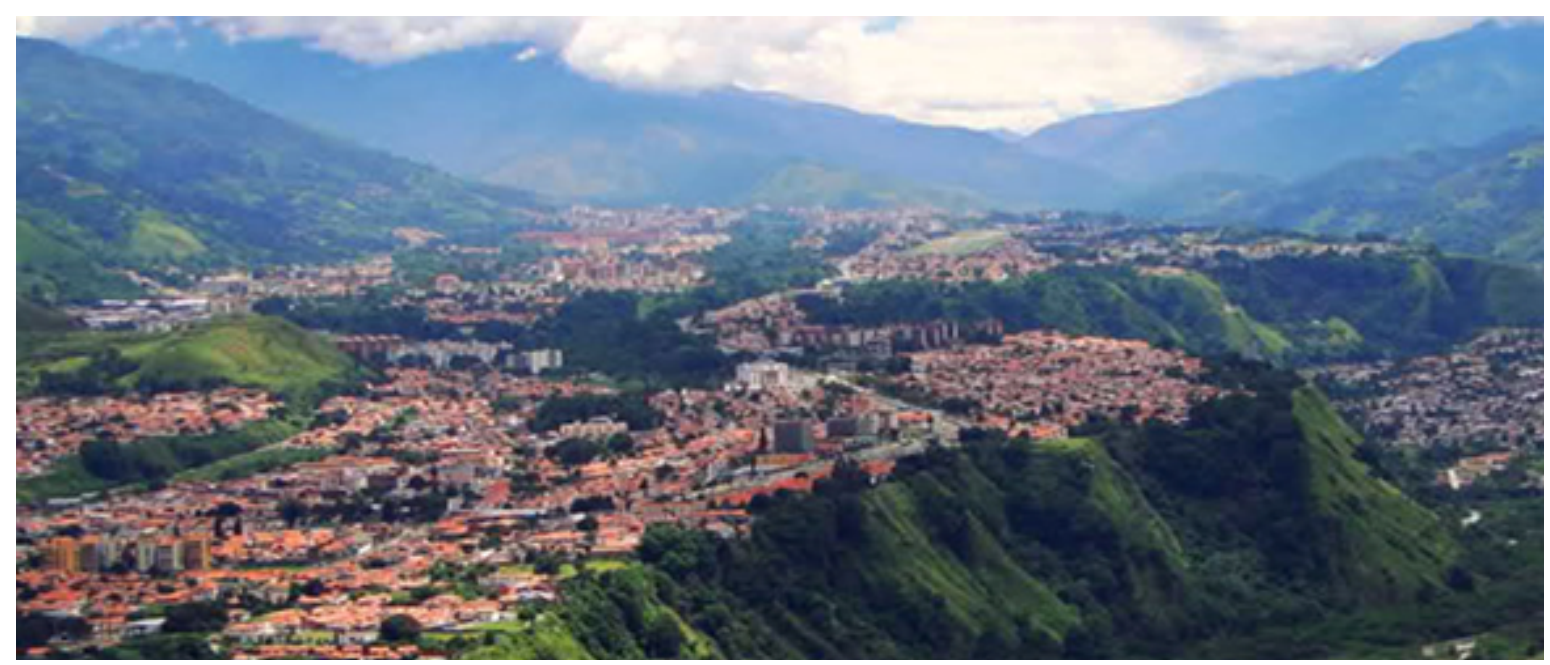

Figura 2. Meseta de la Ciudad de Mérida. Área Metropolitana.

Nota: representa una vista aérea del área metropolitana. Tomado de Wikipedia. 
Mérida, también conocida por ser una ciudad estudiantil, cuenta con la Universidad de los Andes (ULA), alma máter de célebres personajes tanto de la región como del país. La ULA es la Universidad de mayor tradición en el país y la segunda en antigüedad, se ha constituido en el motor de aceleración del desarrollo y de las transformaciones económicas, convirtiéndose en una políitica central prioritaria.

Para el 14 de Junio de 1995 la Ciudad fue decretada Zona Libre cultural, científica y tecnológica a partir de la Gaceta Oficial de la República de Venezuela N 4.937 Extraordinaria y el reglamento de fecha 09 de septiembre de 1998, publicado en la gaceta oficial de la República de Venezuela $N^{\circ}$ 36.611 , régimen fiscal especial de carácter preferencial, establecido con el objeto de producción, divulgación y distribución de bienes y actividades culturales, científicas y tecnológicas en la región.

Según estimaciones del Instituto de Investigaciones Económicas y Sociales (IIES) de la ULA (1996), durante los años 1993-1995, la actividad agrícola contribuyó, entre un 17 y 19\% a la generación del Producto Interno Bruto Total del Estado (PIBEM). Dicha participación es muy superior a la presentada por el sector agrícola en relación con el PIB del país, la cual se ubicaba alrededor del 5-6\% para el período considerado y en el orden del $6-7 \%$ en cuanto a su contribución directa al PIB no petrolero.

La expansión urbana de la periferia ha desbordado los límites administrativos hasta absorber núcleos urbanos adyacentes, iniciando procesos de conurbación, convirtiendo el área de estudio en grandes dimensiones territoriales integradas por múltiples municipios y una intensa actividad en ellas.

\section{MÉTODO}

En esta época, de cambio social tan acentuado, en donde se genera un aumento de las desigualdades junto con una mayor segregación en la ciudad, debemos comprender la ciudad, acompañada de una globalización de valores, en lo que se refiere al medio ambiente, los derechos humanos, las diversidades étnicas y la calidad de vida, tratando de que la sociedad esté consiente de sus derechos; siempre y cuando el desarrollo urbano futuro se base en proyectos de inversiones inmobiliarios, relacionando lo social, con lo espacial, es decir, la política local, regional y micro local, distribuyendo las externalidades, entre el campo y la ciudad o entre ciudad y ciudad.

No son muchos los estudios que a escala internacional o regional se han desarrollado sobre estas áreas, sin embargo, en función de algunas teorías, se plantea la metodología de este trabajo, donde el mismo, consiste en un estudio descriptivo, a través del análisis bibliográfico de las bases teóricas de la ciudad intermedia, junto con las características primordiales propias de este tipo de asentamiento.

Con el fin de comprender la importancia de estos espacios fueron analizadas estadísticas del Programa de las Naciones Unidas para los Asentamientos Humanos (ONU- Habitat), del 2012, donde interpretan que la mayoría de la población urbana mundial (alrededor de un 56\%) vive en ciudades de tamaño medio y pequeño, de menos de 500.000 habitantes, acogiendo aproximadamente $\quad 1.300$ millones de habitantes, siendo, que a través 
de estos centros urbanos pequeños y medianos, la mayoría de la población urbana del planeta y amplios grupos de la población rural pueden acceder a servicios, bienes e infraestructuras, más o menos especializados. Logrando así clasificar a la ciudad de Mérida dentro de los centros descritos bajo estos términos.

Adicionalmente, la ONU-Habitat (2012) afirma que tanto América Latina como el
Caribe son regiones fundamentalmente urbanas, donde casi el 80 \% de la población vive en áreas urbanas, caracterizada por la concentración de la población en unas pocas ciudades, que predominan sobre el resto, sin embargo, son las ciudades intermedias y pequeñas donde reside la mayor parte de la población urbana, generando una oportunidad de un posible reequilibrio de la red de ciudades, en la región y a una mayor diversificación. (Figura 3).

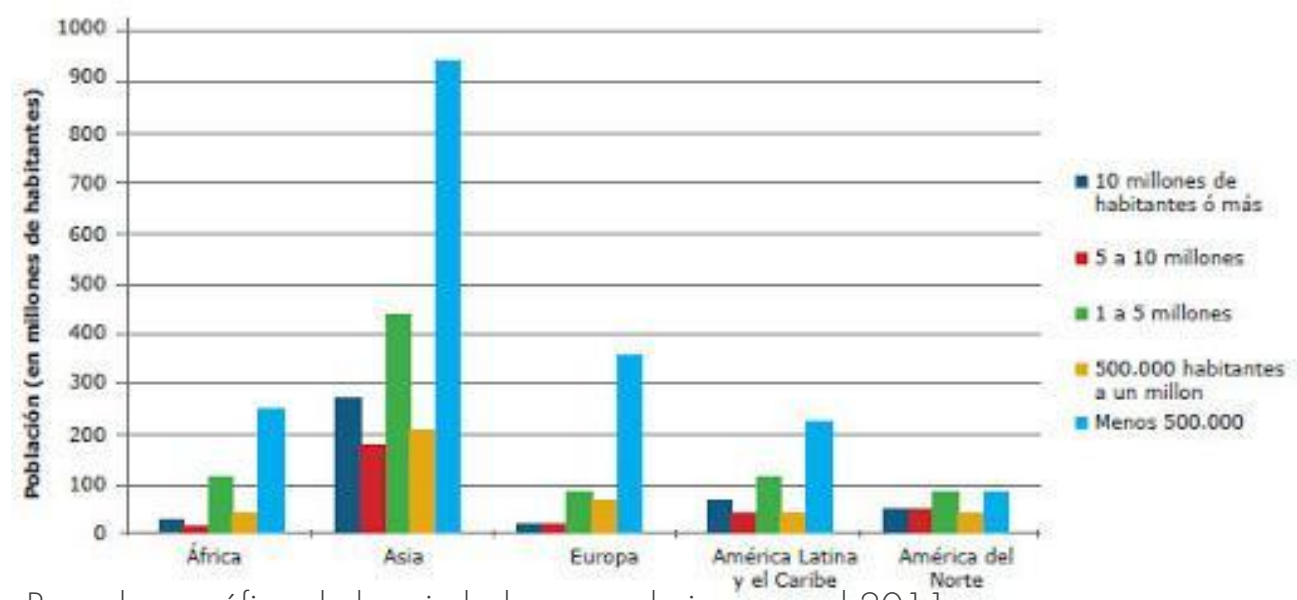

Figura 2. Peso demográfico de las ciudades secundarias para el 2011

Nota: El gráfico representa la población en millones, destacando como la mayor población se localiza sobre las ciudades de menos de 500.000 habitantes, consideradas como intermedias.

Tomado de la Onu Habitat, 2012.

Para una mejor comprensión del término 2. Centros de interacción social, económica y fueron utilizadas las líneas de trabajo del cultural. Son "el corazón económico de programa de la Unión Internacional de amplias áreas rurales en las ciudades del Arquitectos en Barcelona, a través de Bellet Tercer Mundo" o países en vías de desarrollo. y LLop (2007), donde nos definen la ciudad intermedia de acuerdo al rol que juega en su territorio, en una escala local/regional, considerándolas como:

3. Centros ligados a redes de infraestructuras, que conectan las redes locales, y nacionales e incluso, algunas, con fácil acceso a las internacionales lcomo es el caso de las 1.Centros servidores de bienes y servicios ciudades medias, ubicadas en la periferia más o menos especializados, para la metropolitana). Son nodos que articulan flujos, población del mismo municipio y de puntos de referencia y de acceso a otros otrosorganismos locales lasentamientos niveles de la red.

urbanos y rurales), más o menos cercanos sobre los que ejerce cierta influencia.

4. Centros que suelen alojar niveles de la 
administración de gobierno local y población que contiene, dando así origen regional, a través de los cuales se canalizan a las mega ciudades y a las metrópolis, las demandas y necesidades de amplias que corresponden con grandes conurbacapas de la población. ciones, conformadas por la unión de varias áreas metropolitanas o diversas conurba5. Asentamientos con escalas más humanas ciones pequeñas, llamadas ciudades intery aprehensibles, que ayudan al ciudadano medias o medias; aglomeraciones éstas, a identificarse más con su ciudad, teniendo que generan grandes problemas e impacasí una identidad propia launque ello sea tos ambientales y que deberá transformás difícil, en su área de influencia de una marse en grandes retos profesionales en metrópoli). Sin los problemas medioambien- los próximos años del siglo XXI. tales que presentan las megaciudades /p. 5).

Para comprender algunos aspectos se hace necesario entender que la definición Posteriormente, se evaluaron los de ciudad varía según las especificidades, mecanismos de planificación, con el fin de leyes o reglamentos de cada país, asegurar la relación de las áreas urbanas, existiendo ciudades que se centran en junto con las áreas rurales, logrando así la criterios cuantitativos y otras en criterios cohesión territorial, la sostenibilidad en el cualitativos, obteniendo la categorización tiempo y la eficiencia que se busca, de las ciudades en su nación.

herramienta que deberá concretar estrategias eficaces que mantendrán esta En principio, una ciudad intermedia relación, entre el espacio regional y el área corresponde a espacios donde interactúa urbana de una ciudad. En lo que respecta lo urbano con lo rural. Evaluando las al análisis del área de estudio, se evaluaron condiciones entre estos dos términos la condición de ciudad intermedia en urbano y rural, encontramos, que:

función de criterios cuantitativos y cualitativos y la forma en que la institución El área rural, es un espacio que se puede venezolana aborda dichos espacios, para identificar mediante la distribución, de proceder a la selección, análisis y sus elementos en una forma dispersa, conclusión del trabajo.

\section{RESULTADOS LAS CIUDADES INTERMEDIAS.} colmado de tradiciones que marca una identidad, lugar donde se produce el sustento económico, tanto para ellos, como para las áreas urbanas, rodeado de espacios naturales, cuya relación de la población es más directa con el medio La migración que se produce desde las ambiente, espacios que genera una zonas rurales hacia las urbanas, desde la relación más respetuosa con la revolución industrial, ha sido un naturaleza, producción que se realiza a componente importante en el crecimiento y través de la agricultura, el turismo, la el proceso de urbanización de los espacios pesca, la ganadería, la extracción urbanos. Ha incentivado una concentración forestal o la minería, espacios que cubren poblacional que identifica diversos tipos de sus necesidades de servicios, con la ciudades en función de la cantidad de periferia de las ciudades. 
En cuanto al término urbano o de ciudad se puede decir que corresponden a asentamientos densificados, con una mayor concentración de la población, del tipo compacta y dispersa, pero con un porcentaje menor de interacción con el medio ambiente, caracterizado en una forma cultural, con el inicio del desarrollo de actividades complementarias al campo, definidas como actividades de servicios más especializadas, que cumplen las exigencias de la población para el logro de una mejor calidad de vida urbana, caracterizado por una forma de ocupación más territorial, definido principalmente por las funcionalidades urbanas, destacando las actividades económicas y el empleo, como elementos para dinamizar la productividad de los diferentes sectores, lugar donde, se centralizan los gobiernos y los organismos de desarrollo y crecimiento.

Este término de ciudad intermedia no sólo puede ser utilizado para los asentamientos que contienen una ciudad del tamaño medio, pueden ser considerados espacios pertenecientes a las grandes ciudades, llamados espacios periurbanos que, por la cercanía al área rural, presentan condiciones ideales para que ambos espacios interactúen eficientemente

En la gran mayoría de los países de América Latina, a partir de los años 90, el espacio periurbano toma gran importancia, debido a la gran diversidad de actividades desempeñadas por los diferentes agentes socioeconómicos, donde se promueve la expansión territorial, en respuestas a funciones residenciales, de abastecimiento, industriales, sociales de esparcimiento, de recreación y ecológicos.
Carter, en 1974, "sostiene que el periurbano se expande al azar, produciéndose avances significativos y rápidos en algunos sectores y sin progresar nada en otros" (Cardoso y Ortiz, 2008, p. 2). De igual manera, estos procesos, llamados también peri urbanización, se producen -según Cardoso y Ortiz (2008)bajo modelos de expansión urbana, como: a) desarrollo discontinuo (la franja periurbana surge en torno a la ciudad central de manera irregular, dando origen a un área de baja densidad de población); b) desarrollo radial (siguiendo los ejes trazados por las principales vías de acceso a la ciudad) y c) desarrollo a saltos lla peri urbanización actúa como núcleos de usos del suelo urbano en medio de usos exclusivamente rurales) (P. 2).

Esta forma de expansión urbana que se viene dando, es considerada por Vale (2005) como la creadora de los espacios periurbano, es decir, la expansión de la periferia hacia el área de contacto urbano/rural en el interior de las áreas metropolitanas /Cardoso y Ortiz, 2008, p.21. Derivado de esto, Cardoso y Ortiz (2008), consideran que "con el fenómeno de la peri-urbanización, varias actividades que eran exclusivamente urbanas /como centros comerciales, educativos, deportivos, etc.) pasaron a ser poli sectorial, porque actualmente se encuentran en dominios urbanos, rurales y en la franja rur urbana" (p. 3).

Josep María Llop Torné (2013), director de la Cátedra UNESCO de Ciudades Intermedias de la Universidad de Lleida, afirma que "las ciudades son la riqueza de las naciones, pero con el matiz que no pueden cumplir esta función sólo las 
grandes, porque son pocas: es la tarea crucial de una red de urbes intermedias", siendo que, para él, la solución pasaría por crear redes que fomenten una relación entre ciudades, donde unas posean condiciones más uniformes que otras. (p. 34), debiendo comprender, que son estos espacios, los que van a funcionar más equilibrados y sostenibles, que podrán ejercen relaciones más ecuánimes, donde estas se pueden mantener de una forma más armónica con su territorio.

Las ciudades intermedias son centros de interacción social, económica y cultural, características cualitativas con las que se puede definir la ciudad de tamaño medio. Centros de intercambio de bienes y servicios, tanto para la población de la ciudad, como para sus espacios aledaños, áreas que generan focos de articulación y de conexión territorial, donde sus dinámicas socio económicos son determinadas por su ubicación.

Estas áreas fomentan desplazamientos saludables y sustentables, con calidad de vida, pudiendo localizar los equipamientos y servicios básicos que requiere la ciudad. Provocando a la vez una relación más cercana de los ciudadanos, con los espacios comunitarios, de servicios y recreativos, obteniendo una apropiación colectiva del territorio, situación que permite conformar una identidad local y un arraigo cultural y patrimonial.

En el Foro de Cooperación Sur-Sur se utiliza un concepto flexible de ciudad intermedia, basada en la población /con menos de un millón de habitantes y más de 50.0001 y de su rol en la mediación de flujos de mercancías, información, innovaciones y administración, entre los territorios rurales y urbanos, dentro de sus áreas de influencia y respecto a otras ciudades y regiones (2016, p. 2), donde se plantea una definición de ciudad intermedia mucho más amplia. En esta, se considera que las ciudades intermedias son urbes creadoras de puentes de conexión importantes entre zonas rurales y urbanas.

De este modo, para la población rural, este tipo de ciudades constituyen también una oportunidad para acceder a instalaciones básicas (como escuelas, hospitales, administración, mercados) y también servicios (como el empleo, la electricidad, los servicios de tecnología, transporte). Al tener esta posición intermedia, también son, para la mayoría de los ciudadanos, un foco de transición para salir de la pobreza rural. Además, las relaciones que crean con las grandes urbes les permiten complementarse entre ciudades y funcionar como un sistema de ciudades (Roig, 2014, p. 44-46), espacios donde se obtienen beneficios locales, dentro de la ciudad y establecen una mejor función con otras ciudades.

Es de hacer notar que, al poseer espacios intermedios, podremos conectarnos con territorios rurales cercanos, estimulando un equilibrio entre el asentamiento y la naturaleza, generando sinergia, potenciando la asociación, la productividad y la diversificación de la económica, estrategias que podrían verse como una forma más eficiente de ordenar las grandes aglomeraciones urbanas, considerando que en la actualidad a los municipios se les hace difícil, específicamente, controlar el desarrollo de 
las áreas periféricas de esos espacios y entender la relación con las áreas rurales. La posibilidad de articular el territorio implica de metodologías y conceptos claros y fundamentales, con enfoques dirigidos hacia la conformación de un equilibrio entre lo rural y lo urbano, que lleven al territorio a un futuro deseado.

Para ello, es necesario que este proceso se enfatice en un método continuo, el cual, de acuerdo con Soms (1995), funciona como un conjunto de procedimientos para relacionar lo que se quiere lograr (objetivos), con la forma de lograrlo (estrategias) y los instrumentos de que se disponen para tales propósitos (políticas, programas, presupuestos). En este sentido, la planificación es un ejercicio permanente e iterativo, en tanto desarrolla sus planteamientos y propuestas a través de sucesivas aproximaciones, proponiendo, verificando y retroalimentando las proposiciones iniciales (Sandoval, 2014, p. 121.

\section{EL ÁREA METROPOLITANA DE MÉRIDA, UNA CIUDAD INTERMEDIA}

Según criterios cuantitativos, la ciudad de Mérida opta para calificar como una ciudad intermedia: en su último censo elaborado (Instituto Nacional de Estadística y Geografía [INEGI], 2000), la población total corresponde a 508.988 habitantes, información que la ubica entre el rango de 500.000 a 1.000 .000 habitantes.

En cuanto al criterio cualitativo, utilizaremos aspectos tomados de Bellet y Llop (2007, p. 5), donde se comparó desde el punto de vista del papel o la función que la ciudad juega en su territorio; influencia y relación que ejerce y mantiene dentro de este espacio; flujos y relaciones que genera hacia el exterior, junto con el rol que juega la ciudad de Mérida dentro de su territorio:

1.El área metropolitana de Mérida corresponde a un centro de servicios más o menos especializados, ejerciendo influencia sobre los municipios aledaños a la urbe, esta área despliega relaciones con todos los municipios del Estado, ya que administrativamente así está conformado, por un gobernador que interactúa con los alcaldes y administradores de los municipios insertos en el Estado Mérida, trabajando coordinadamente tanto en el turismo, como en las actividades productoras específicas de cada sector.

2. Corresponde a un centro de interacción social, económica y cultural, tanto por su actividad económica diversificada que posee, como por el servicio estudiantil y turístico, que interactúa con sus 23 municipios, influenciado por sectores como son Educacional y socio-cultural.

3.Debido a la importancia de su infraestructura y al mundo globalizado, la ciudad conecta con redes locales, regionales, nacionales e internacionales.

4.Debido a la proclamación como zonas libre cultural, científica y tecnológica, Mérida tiene como estrategia, ser centros de innovación y desarrollo tecnológico, proporcionando ambientes que incentiven la creatividad y el intercambio, a través de equipamiento e infraestructura de carácter internacional: culturales, científicos y deportivos, entre otros. 
De lo cual, podemos afirmar que, tanto desde el punto de vista cuantitativo como cualitativo, la ciudad puede ser considerada como intermedia, reflejando diversas fortalezas que ayudarían al sector en desarrollar ambos espacios, donde su centro urbano y los espacios colindantes generaren focos de articulación y de conexión territorial, fomentando desplazamientos saludables, eficientes y sustentables.

Unas de las grandes debilidades que podemos destacar, en el sistema de planificación de Venezuela, tiene que ver con un hecho administrativo que separa en materia de planificación a organismos responsables de las áreas, según las funciones que cada institución tiene establecida, situación necesaria de evaluar para el resultado eficiente del trabajo.

\section{LA PLANIFICACIÓN ESTRATÉGICA: UNA OPCIÓN DE DESARROLLO PARA EL ÁREA METROPOLITANA DE MÉRIDA.}

La planificación estratégica nace de la necesidad de perfeccionar la planificación normativa, permite en su aplicación adaptarse a las realidades territoriales, a las diferentes técnicas, para facilitar una rápida apropiación por parte de los involucrados, escogiendo la opción más viable y efectiva a la hora de que ocurran las situaciones. Esta metodología surge de la necesidad de poseer una herramienta que logre el desarrollo local, dentro de un territorio (Sandoval, 2014, p. 12).

Todo instrumento de planificación debe consistir en un proceso de organización, que relacione y modifique la estructura $e$ interrelaciones presentes a nivel regional o local, generando dinámicas en donde el objeto debe ser modificado antes, durante y después del proceso de una estructura social asociada a un territorio.

Se plantea, entonces, para el área Metropolitana de Mérida, la planificación territorial estratégica u ordenamiento territorial, disciplina que se asocia más a la relación de lo urbano con lo rural, metodología acertada para las ciudades intermedias, quienes se caracterizan por una mejor conexión territorial, minimizando los efectos de la intervención humana en el territorio, en donde se analice, desarrolle y gestione estos espacios geográficos urbanos y rurales, potenciando e integrando características físicas o naturales, condiciones ambientales, económicas, políticas, sociales y culturales, propiciando el desarrollo sostenible, incluyendo la planificación del uso del suelo, la planificación urbana, la regional, la gestión ambiental, la geografía humana y la economía.

De la planificación territorial estratégica se logra obtener el instrumento que regula el uso del territorio, junto con las mejores opciones - respuestas territoriales representadas en estrategias acordes con el entorno, colaborando con las organizaciones a enfrentar los problemas que se pueden generar durante una gestión, logrando así prevenir y no sólo predecir, es decir, consiste en la integración o la esencia de una planificación normativa junto a la planificación situacional o estratégica.

$\mathrm{Si}$ visualizamos las condiciones que caracterizan las áreas urbanas y las rurales del área de estudio, podemos encontrar que, en las zonas rurales, predomina el verde sobre lo construido, con una 
localización dispersa de sus elementos, contrario a lo urbano, donde lo construido priva sobre el verde, con un desarrollo más compacto. A fin de lograr la cohesión territorial que se busca, cada una de las partes de la ciudad deben interactuar y entrar en equilibrio. Es así, como el mecanismo de planificación implementado para el área de estudio deberá tomar en consideración elementos físicos, tales como, el porcentaje del verde, con respecto al porcentaje de elementos construido, además, del reordenamiento de sus usos, sin llegar a disminuir las condiciones de calidad ambiental que poseen las áreas rurales. Se trata de una forma de interacción más eficiente.

Considerando que la calidad de vida es el equilibrio entre la calidad ambiental, la identidad cultural y el bienestar, se puede decir que el desarrollo urbano se encuentra en medio de estos tres aspectos, donde se establecen condiciones para lograr el bienestar del individuo $y$, por ende, la calidad de vida, donde se logre un proceso técnicamente posible, económicamente viable, socio-culturalmente aceptable y ambientalmente adaptable, virtudes que podemos encontrar tanto en las áreas urbanas como en las rurales del área de estudio.

En ellas, se aspira conseguir espacios definidos con identidad, donde política y socialmente se encuentren integradas y diferenciadas a la vez, es decir, una verdadera cohesión social y territorial, donde se logre optimizar el uso y la conformación de un desarrollo sustentable. Para que el instrumento logre obtener la imagen de ciudad deseada, se hace indispensable elaborar una planificación integral de ambos indistintamente del organismo que lo dirija, situación que creara un sentido compartido y colectivo de sus ocupantes y de las organizaciones, junto a la imagen deseada. Es entonces, la planificación territorial estratégica, el instrumento que logrará regular el territorio urbano y rural del área de estudio, mediante la definición de estrategias acorde con la realidad y el mecanismo de evaluación del instrumento, logrando así dar soluciones de forma integral, con las características tan particulares que cada espacio posee, conformando centralidades autoabastecidas e independientes, proporcionando los servicios básicos que requiere, disminuyendo o limitando el crecimiento acelerado que las ciudades llevan en la actualidad, obteniendo como resultado una visión diferente del tipo de urbanización a desarrollar.

\section{CONCLUSIÓN}

Las áreas rurales y urbanas por lo general son analizadas en forma separada, aun sabiendo que poseen problemas universales con características comunes, ya sea por las externalidades y la globalización, problemas que ambos espacios generan debido a la desconexión o el desequilibrio entre la humanidad y el resto de la naturaleza, lo que hace que aumente la brecha metabólica, es decir, que se produzca la división entre la ciudad y el campo.

Es la planificación Territorial estratégica u de ordenamiento territorial, junto a la actual tecnología, donde se generará una oportunidad para articular las relaciones entre lo rural y lo urbano, en el área de estudio, desde lo local y lo regional, donde se producirá una oportunidad que tendría como objetivo mejorar la calidad de vida de 
ambos sectores, mediante la generación de empleo, la disminución de la contaminación que genera esos espacios, la utilización eficiente de los recursos y la construcción de un hábitat planificado, de acuerdo a las condiciones organizacionales de cada espacio /compacto en la ciudad y disperso en lo rural).

Derivado de esto se propone, para el área de estudio, un ordenamiento territorial que articule el campo y la ciudad, con una mejor distribución de la población en donde se produzca el equilibrio que se busca en la sostenibilidad, estableciendo una amigable relación de su población con el medio ambiente, es decir, una mejor y más sostenible distribución de su población en todo el territorio.

El deterioro del medio ambiente parece ser una consecuencia directa de los procesos de globalización, vinculado a la cuestión de ciudad y la cuestión de lo local, provocándose de esta manera posturas eclécticas y moderadas, razón por la cual el administrador y el planificador de un municipio y en espacial del área de estudio deben entender ambas posiciones, al comprender que son espacios que van a funcionar más equilibrados y sostenibles, que podrán ejercer relaciones más ecuánimes, manteniéndolas de una forma más armónicas y que generaran relaciones abiertas y equilibradas en su territorio.

Para concretar parámetros por los cuales podemos identificar la ciudad intermedia, basados en criterios cuantitativos y cualitativos, podemos establecer que cuantitativamente una ciudad intermedia se ubica entre 2.500 y 1.000 .000 de habitantes, considerando que cualitativamente debe facilitar los flujos y relaciones, generando equilibrios con el exterior, en aspectos culturales, sociales, políticos, económicos y ambientales, es decir, serán intermedias aquellas ciudades que cumplan con esta condición demográfica y con las dinámicas relacionadas en las diferentes escalas territoriales, considerando que dentro de una Metrópolis podremos identificar la ciudad intermedia a aquellos espacios que se localizan en la periferia, donde obtendremos la relación directa del campo y la ciudad.

Para lograr un desarrollo sostenible del área de estudio, es entonces necesario:

- Un ordenamiento territorial y estratégico, como proyecto o programa de ciudad, a largo y medio plazo (estrategia).

- Una planificación física o urbanística, más coherente con el tamaño de las ciudades intermedias (escala urbana, diseño urbano), en donde bajo una imagen de ciudad se logre establecer un equilibrio social, económico y sobre todo ambiental, con la comparación entre el verde y lo construido, como elementos adaptados al territorio físico y al entorno natural de la ciudad, para lograr el urbanismo sostenible.

- Definir estrategias que sean sostenidas en el tiempo y cambiables, según la verificación y retroalimentación las mismas.

- Una planificación que integre el entorno territorial, con menos polución, espacios públicos eficientes, menor consumo energético, con mejor movilidad, es decir, una ciudad sostenible, con mejor metabolismo.

De esta manera, obtendremos puentes de conexión entre las zonas rurales y urbanas, 
oportunidad para ambos espacios de Las ciudades intermedias sin planificación complementarse con las fortalezas y son espacios que, por lo general, generan oportunidades, que cada uno tiene, para así grandes problemas e impactos disminuir debilidades y aliviar las amenazas ambientales, razón por la cual debemos de una forma conjunta.

En definitiva, la administración de la ciudad de Mérida debe considerar una planificación territorial y estratégica, que contrarreste retos de equidad (referidos a la igualdad entre el campo y la ciudad), reto territorial len relación a la cohesión territorial, considerando ventajas y desventajas de la ciudad), reto climático (en relación a los cambios hacia la sostenibilidad para contrarrestar las transformaciones ambientales que se están dando) y el reto de participación ciudadana la fin de lograr la incorporación plena de la ciudadanía en la determinación, formulación, monitoreo y vigilancia de las políticas públicas), diagnosticados a través del estudio de las Fortalezas, debilidades, amenazas y oportunidades, complementando estrategias ante situaciones inesperadas, en una forma viable y efectiva.

Para la integración de ambos espacios, tanto de la ciudad como del campo, se propone comparar su equilibrio a través del porcentaje de conexión que posea con el territorio, por ejemplo, si evaluamos el equilibrio ambiental, de un sector rural, podremos identificar el porcentaje de verde y el de construido. En la búsqueda de que entren en equilibrio con la ciudad podemos transformarla bajo un desarrollo que utilice el factor inversa de estos datos, con la finalidad de evitar que la ciudad deje de ser un espacio intermedio y pierda su condición funcional con el entorno. pensarlo como grandes retos, considerando que la gran mayoría de la población mundial vive en centros urbanos de tamaño medio, siendo áreas donde la población tanto urbana como rural podrían acceder a servicios, bienes e infraestructura más especializados, colocando este instrumento como acción prioritaria, para el ordenamiento y mejoramiento de la calidad de vida, logrando así que sus habitantes tengan la oportunidad de optar por vivir en cualquiera de estos espacios, sin necesidad de tener que emigrar, en búsqueda de servicios básicos, para una mejor calidad de vida, aspecto fundamental con el cual podremos revertir el efecto provocado por la forma de urbanización que se originó después de la revolución industrial.

Hoy en día, las poblaciones urbanas están aumentando a un ritmo acelerado, la ciudad de Mérida no se encuentra exenta de este proceso, incrementando al mismo tiempo la huella ecológica. Ante esta situación y el calentamiento global se hace necesario un cambio de conciencia y de paradigma. Para ello, las ciudades intermedias tienen las condiciones ideales de construir territorios sostenibles, con mayor capacidad, mejor calidad de vida y mayores oportunidades de orientarnos a la sostenibilidad, a través de una mejor comprensión del medio ambiente natural, es decir, una manera de relacionar a la población con cada uno de los elementos de la ciudad. 


\section{REFERENCIAS BIBLIOGRÁFICAS}

Bellet S. C., y Llop T. J. (2007). Miradas a otros Espacios Urbanos: Las Ciudades Intermedias. Scripta Nova: Revista Electrónica de Geografía y Ciencias Sociales, 8(165), p. 1-28. http://hdl.handle.net/10459.1/41650

Cardozo, A. y Ortiz, J. (2008). Periurbanización, segregación y fragmentación territorial. Institutos de Estudios Geográficos de Universidad Nacional de Tucumán. http:/ / observatoriogeograficoamericalatin a. org. m x / e gal l 2/. Geografiasocioeconomica/Geografiaurba na/255.pdf

Colina, J., Volcanes, V., Milgreya, C., Pinto, F., Salas, M., Klarica, S., y Sosa, G. (2014). Determinación de las velocidades de corte en los primeros $30 \mathrm{~m}$ de profundidad a partir de la refracción de microtremores ReMi en el Área Metropolitana de Mérida. GEOS. Revista Venezolana de Ciencias de la Tierra, (46), 18-26. http: //saber.ucv.ve/ojs/index.php/rev_geos/article/view/11803

Foro de Cooperación Sur-Sur y Triangular. (2016). Políticas para el desarrollo inclusivo en ciudades intermedias y gobiernos regionales, Santa Fe, Argentina. https://www.uclg.org/sites/default/files/ programa_foro_cglu_oit_gsf.pdfInstituto Nacional de Estadística y Geografía. (2000). XII Censo General de Población y Vivienda 2000.

Llop, J. (2013) La dimensión urbana adecuada para el desarrollo sostenible/Entrevistador: A. Punsola. Organización Ciudades y Gobiernos Locales Unidos. https://www.uclg.org/sites/default/files/news_i_cities.pdf

Programa de las Naciones Unidas para los Asentamientos Humanos. (2012). Estado de las Ciudades de América Latina y el Caribe 2012. Rumbo a una nueva transición urbana. http://www.zaragoza.es/contenidos/medioambiente/onu/newsletter12/887_spa.pdf

Roig, J. (2014). Las ciudades intermedias en la nueva agenda urbana. Revista Ciudad Sostenible, 18, 44-46.

Sandoval, C. (2014). Métodos y aplicaciones de la planificación regional y local en América Latina. CEPAL- Serie Desarrollo Territorial (17). Naciones Unidas. http://hdl.handle.net/1 $1362 / 36967$ 